\title{
Reproductive Potential of Monosporascus cannonballus
}

\author{
M. M. Waugh, D. H. Kim, D. M. Ferrin, and M. E. Stanghellini, Department of Plant Pathology, University of \\ California, Riverside 92521
}

\begin{abstract}
Waugh, M. M., Kim, D. H., Ferrin, D. M., and Stanghellini, M. E. 2003. Reproductive potential of Monosporascus cannonballus. Plant Dis. 87:45-50.

Vine decline of melons caused by Monosporascus cannonballus is a destructive disease worldwide. Ascospores, the only spore stage produced by this soilborne fungus, serve as the primary inoculum. Ascospore production on roots occurs primarily at the end of the cropping season, and high soil temperatures $\left(25\right.$ to $\left.30^{\circ} \mathrm{C}\right)$ govern, in part, the rate of reproduction of the pathogen. In vitro studies confirm that the optimal temperature for reproduction ranged from 25 to $30^{\circ} \mathrm{C}$. Additionally, the root system of a single mature cantaloupe plant is capable of supporting the production of approximately 400,000 ascospores. The latter population, if incorporated uniformly into $0.03 \mathrm{~m}^{3}\left(1 \mathrm{ft}^{3}\right)$ of soil, would be equivalent to 10 ascospores per gram of soil. Known problem fields contain as few as 2 ascospores per gram of soil. These results offer a possible explanation for field observations that economically significant disease problems can occur after only two consecutive melon crops if environmental conditions are conducive to pathogen reproduction, and they suggest that strategies to inhibit reproduction would be instrumental in disease management.
\end{abstract}

Monosporascus cannonballus Pollack \& Uecker, a root-infecting ascomycete, is the causal agent of a destructive vine decline of cantaloupe (Cucumis melo L.) worldwide $(1,5)$. The disease is characterized by the sudden and generally uniform collapse (wilt) of entire fields, which commonly occurs within 2 weeks of harvest.

Ascospores, which are produced in perithecia on infected roots, function as the primary survival structure and inoculum for root infection $(10,11)$. Although the fungus can infect melon roots early in the growing season (i.e., 9 days after planting, M. E. Stanghellini, unpublished), pathogen reproduction has been observed to occur late in the growing season $(3,4,7)$. However, no quantitative data exist regarding pathogen reproduction either in the field or in the laboratory. Knowledge of the factors influencing the reproductive phase of this pathogen could be instrumental in the development of strategies for disease management. As pointed out by Menzies (6), "Factors associated with the initial production of inoculum are just as important as factors affecting later survival and they may be easier to apply in population control."

Our objectives were to document the rate of reproduction as well as the reproductive potential of the pathogen on in-

Corresponding author: M. E. Stanghellini

E-mail: michael.stanghellini@ucr.edu

Accepted for publication 19 August 2002.

Publication no. D-2002-1119-02R

(C) 2003 The American Phytopathological Society fected cantaloupe roots under field conditions. Additionally, we wanted to assess the influence of temperature and nutrition on the in vitro vegetative growth and reproduction of $M$. cannonballus. A preliminary report has been published (14).

\section{MATERIALS AND METHODS}

Pathogen reproduction on cantaloupe roots was monitored in four fields at the end of two spring and two fall cropping seasons. All fields had a known history and uniform distribution of disease. In 2000, the spring melon crop was seeded in April and terminated in the middle of June; in 2001, it was seeded in May and terminated in the middle of July. The fall crops in 1999 and 2000 were both planted in August and were terminated at the beginning of December and the middle of November, respectively.

Spring field studies. All three spring field studies were conducted at the University of California, Desert Research and Extension Center (DREC) in Holtville, CA.

Ascospore population densities of $M$. cannonballus were estimated utilizing the method described by Stanghellini and Rasmussen (13). The resident population densities of $M$. cannonballus in the naturally infested, 1-ha field sites ranged from 1.4 to 2.9 ascospores per gram of soil (Meloland sandy loam). Seeds of a susceptible cantaloupe cultivar (Caravelle) were sown into the center of raised beds using a spacing of $30.5 \mathrm{~cm}$. Each bed was prepared by installing a drip irrigation line (T-tape with 20.3-cm spacing between emitters delivering 0.025 liters $/ \mathrm{min}$ at $8 \mathrm{psi}$ ) at the 20-cm depth. Beds were $33 \mathrm{~m}$ long, 137 cm wide, and on 203-cm centers. Standard local cultural practices were employed for insect control, fertilization, irrigation frequency, and weed management.

Pathogen reproduction on colonized cantaloupe roots was monitored in two fields in 2000 and one field in 2001, beginning at or just prior to crop termination (i.e., canopy collapse) and continuing at weekly to biweekly intervals.

Sampled plants were chosen arbitrarily from within a 0.5 -ha site within each field, and the root systems of 10 to 15 plants were carefully excavated from soil and gently washed. The total number of roots and the number of roots bearing perithecia of $M$. cannonballus were enumerated for each plant. Roots less than $0.5 \mathrm{~mm}$ in diameter were not included in the studies because preliminary studies indicated that these small roots bear relatively few perithecia.

Fall field studies. Fall field studies were conducted in two drip-irrigated commercial fields in Winterhaven, CA. Seeds of a susceptible cantaloupe cultivar (Primo) were sown into the center of raised beds. All fields had a known history and uniform distribution of the disease. Ascospore population densities in these fields ranged from 0.84 to 1.4 ascospores per gram of soil (Rositas fine sand).

Pathogen reproduction on colonized cantaloupe roots was monitored in one field in 1999 and three fields in 2000, beginning 1 week after the first harvest and continuing biweekly until the grower plowed the fields. No canopy collapse occurred during the fall cropping seasons.

Sampled plants were chosen arbitrarily within a subplot of the fields that consisted of two rows, each $100 \mathrm{~m}$ in length. The root systems of 10 plants were carefully excavated from soil and washed gently. The total number of roots and the number of roots bearing perithecia of $M$. cannonballus were enumerated for each plant.

Soil temperatures. Soil temperatures were recorded using $\mathrm{HOBO}$ data loggers (Model H08-001-02, Onset Computer Corporation, MA). Data loggers were buried between adjacent cantaloupe plants at the 10 -cm soil depth. One data logger per field was used.

In vivo reproductive capacity. Two different methods were employed to quantify the number of ascospores that could be produced on the root system of a naturally infected cantaloupe plant.

First, the root systems of 18 mature cantaloupe plants were carefully excavated 

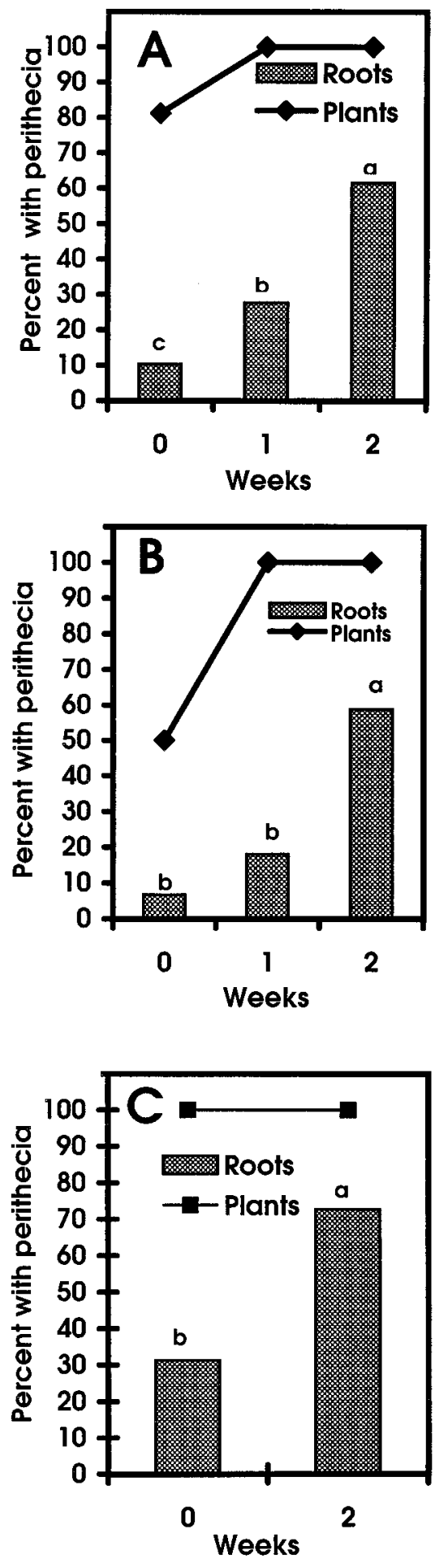

Fig. 1. Perithecial development of Monosporascus cannonballus on naturally infected cantaloupe roots subsequent to canopy collapse at the end of the spring cropping season. A, field 1, spring 2000. B, field 2, spring 2000. C, field 3, spring 2001. Each bar represents the mean of 15 (field 1) or 10 (fields 2 and 3 ) plants. Bars with the same letter do not differ significantly according to Duncan's multiple range test $(P=$ $0.0001)$. Week 0 corresponded to the canopy collapse for fields 1 and 3. For field 2, week 0 corresponded to 1 week prior to the canopy collapse. from soil at the end of both the 1999 and 2000 spring cropping seasons. The length and mean diameter of each root per plant was recorded. Root segments bearing mature perithecia were then excised and cut into 1-cm-long segments, their diameters were measured, and the number of perithecia on each segment was enumerated with the aid of a dissecting microscope. Additionally, 100 perithecia were randomly excised from these root segments, placed on glass slides, and the number of ascospores per perithecia was enumerated with the aid of a compound microscope $(\times 10$ magnification). The above data were then used (i) to determine the relationship between root diameter and number of perithecia, and (ii) to estimate the theoretical number of ascospores that could be produced on a fully colonized root system of a single cantaloupe plant.

Second, the root systems of four naturally infected mature cantaloupe plants were excavated from soil 14 days after plant death. All of the roots on each of the plants had perithecia on $100 \%$ of their length (excluding the taproot). Roots from each plant were severed from the taproot, and their lengths were measured. Roots were then placed in glass beakers containing $200 \mathrm{ml}$ of water and 75, 4-mmdiameter glass beads. The mixture was agitated vigorously with a magnetic stir bar to facilitate release of the ascospores from the perithecia and passed through nested 150-, 74-, and 25- $\mu \mathrm{m}$ sieves. Ascospores retained on the $25-\mu \mathrm{m}$ sieve were then back-washed into a beaker containing a final volume of $140 \mathrm{ml}$ of a $50 \%$ sucrose solution and thoroughly mixed. The ascospore population in each beaker was then estimated by counting, using a dissecting microscope, the number of ascospores in $20,10-\mu \mathrm{l}$ aliquots of the suspension.

Effects of temperature and nutrients on mycelial growth and reproduction in vitro. A culture of $M$. cannonballus (Imp 4-4), isolated from an infected cantaloupe root from the Imperial Valley, CA, was maintained on $10 \%$ V8 juice agar. A $0.5-$ mm-diameter disk of the fungus from a 10day-old culture was placed at the perimeter of a 9-cm-diameter petri plate containing $0.5,2.5,5.0$, or $10 \%$ V8 juice agar. Three plates of each medium were incubated at $20,25,30$, and $35^{\circ} \mathrm{C}$ in the dark. At 24-h intervals, the radial growth of the developing colony was marked, and the mean of three consecutive measurements per plate was used to estimate the daily growth rates at the various temperatures and nutrient concentrations. The experiment was repeated once.

The number of mature perithecia per plate was determined weekly over a 6week period. Perithecia were considered mature when they appeared black in color. The total number of perithecia in five separate areas (each $1.54 \mathrm{~cm}^{2}$, total area 7.7 $\mathrm{cm}^{2}$ ) per plate were enumerated. A tem- plate was used to maintain uniformity of sampling areas from replicate plates. The experiment was terminated when there was no significant increase in the number of perithecia per unit area for at least 2 weeks. All readings were terminated after 42 days. The experiment was repeated once.

Statistical analyses. Each in vitro experiment was repeated once, and the combined data from the two experiments were analyzed by analysis of variance (ANOVA) to determine treatment effects; mean comparisons were made using Duncan's multiple range test. Regression analysis was used to determine the relationship between nutrient concentration, mycelial growth, and perithecia production. Regression analysis was also used to determine the relationship between root diameter and the maximum number of perithecia produced on roots. For the rate of perithecial development, the data were analyzed by ANOVA and the means were separated by treatment and date using Duncan's multiple range test. Analyses were conducted using SAS/STAT software for IBM-PC.

\section{RESULTS}

Spring field studies. At termination (i.e., canopy collapse) of the spring planted melon crops, the percentage of plants with one or more roots bearing perithecia ranged from 81.3 to $100 \%$, and the percentage of roots per plant bearing perithecia ranged from 6.7 to $31.3 \%$ (Fig. 1). When the plants were allowed to remain undisturbed in the field after crop termination (a common grower practice), the percentage of roots per plant bearing perithecia increased significantly (ranging from 58 to $72 \%$ ) within the next 7 to 14 days. Soil temperatures during the last 3 weeks of the 2001 spring cropping season (9 to 24 July 2001), which were similar to those in 2000 , ranged from a low of $26.0^{\circ} \mathrm{C}$ to a high of $33.6^{\circ} \mathrm{C}$ at the $10-\mathrm{cm}$ depth.

Fall field studies. No canopy collapse occurred during the fall cropping seasons. Thus, collection of data on the percentage of plants and roots bearing perithecia was initiated 1 week after the first harvest date and continued at 14-day intervals until the crop was destroyed (plowed) by the grower (Fig. 2). At the first sampling date of both 1999 and 2000, $70 \%$ of the plants had one or more roots bearing perithecia, and the percentage of roots per plant bearing perithecia ranged from 10.4 to $18.3 \%$. As the harvest season progressed, significant increases in the number of roots per plant with perithecia were recorded only in 1999 (Fig. 2A). No increase was recorded in 2000 (Fig. 2B). Soil temperatures during the fall harvest periods are presented in Figure 3 . The temperatures ranged from 15.2 to $24.8^{\circ} \mathrm{C}$ and 11.7 to $20.4^{\circ} \mathrm{C}$, respectively, in 1999 and 2000.

In vivo perithecia production. The mean root length of 36 mature cantaloupe 
plants was $132.1 \pm 7.1 \mathrm{~cm}$, and the mean root diameter was $1.4 \pm 0.1 \mathrm{~mm}$. From these plants, 114 root segments (each $1 \mathrm{~cm}$ long) bearing perithecia were excised, and the number of perithecia on each segment was recorded. There was a significant linear relationship between root diameter and the number of perithecia per $\mathrm{cm}$ root length $\left(P<0.0001 ; R^{2}=0.92\right)$ (Fig. 4). The mean number of ascospores per perithecia, estimated from 100 naturally produced perithecia excised from the above roots, was $66.5 \pm 2.7$. Thus, we estimated that the theoretical number of ascospores that could be produced on the root system of a single cantaloupe plant $(132.1 \mathrm{~cm} \times 44.4$ perithe$\mathrm{cia} / \mathrm{cm} \times 66.5$ ascospores/perithecia) would be 390,039 ascospores.

The latter theoretical number is within the range $(266,000$ to 518,000 ; mean of $395,000 \pm 66,989)$ of actual numbers of ascospores released from the roots of four
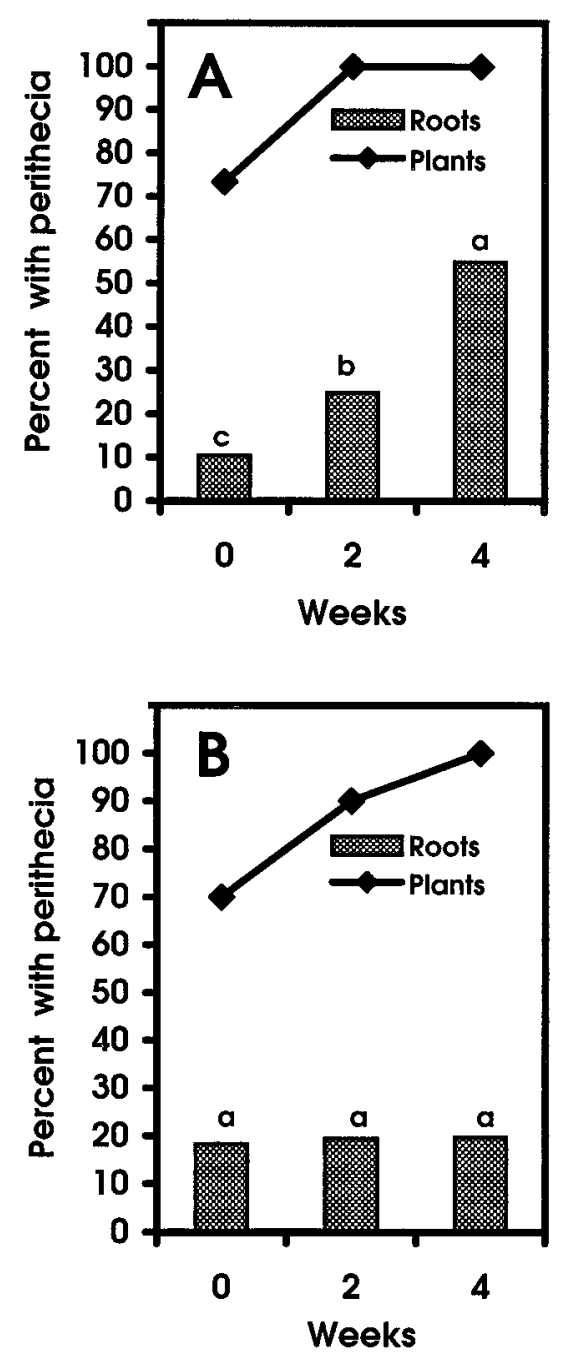

Fig. 2. Perithecial development of Monosporascus cannonballus on naturally infected cantaloupe roots at the end of the fall cropping season. A, field 1, 1999. B, field 2, 2000. Bars with the same letter do not differ significantly according to Duncan's multiple range test $(P=$ $0.0001)$. The studies began 1 week after the first harvest date, week 0 . naturally infected plants excavated from soil 2 weeks after crop termination (Table $1)$.

Effects of temperature and nutrients on mycelial growth in vitro. The effect of temperature on the rate of mycelial growth of $M$. cannonballus at various concentrations of V8 juice agar is presented in Figure 5. Irrespective of the nutrient concentration in the media, the fungus grew significantly faster at $30^{\circ} \mathrm{C}$ than at 20,25 , or $35^{\circ} \mathrm{C}(P=0.0001)$ (Fig. 5A). There was little or no significant difference between mycelial growth rates at 25 and $35^{\circ} \mathrm{C}$. The fungus grew poorly at $20^{\circ} \mathrm{C}$. There was a positive linear relationship between nutrient concentration and mycelial growth at 25,30 , and $35^{\circ} \mathrm{C}\left(P<0.05, R^{2}>0.9\right)$. Regression analyses for all temperature data exhibited the same trend; thus only the data for $30^{\circ} \mathrm{C}$ are presented $\left(P=0.008, R^{2}=\right.$ 0.98) (Fig. 5B).

Effects of temperature and nutrients on reproduction in vitro. The first mature perithecia formed between 14 and 20 days after incubation and reached maximum numbers by day 27 . The same trend was observed with all nutrient concentrations; therefore, only the data from $10 \% \mathrm{~V} 8$ juice agar are presented (Fig. 6). Maximum

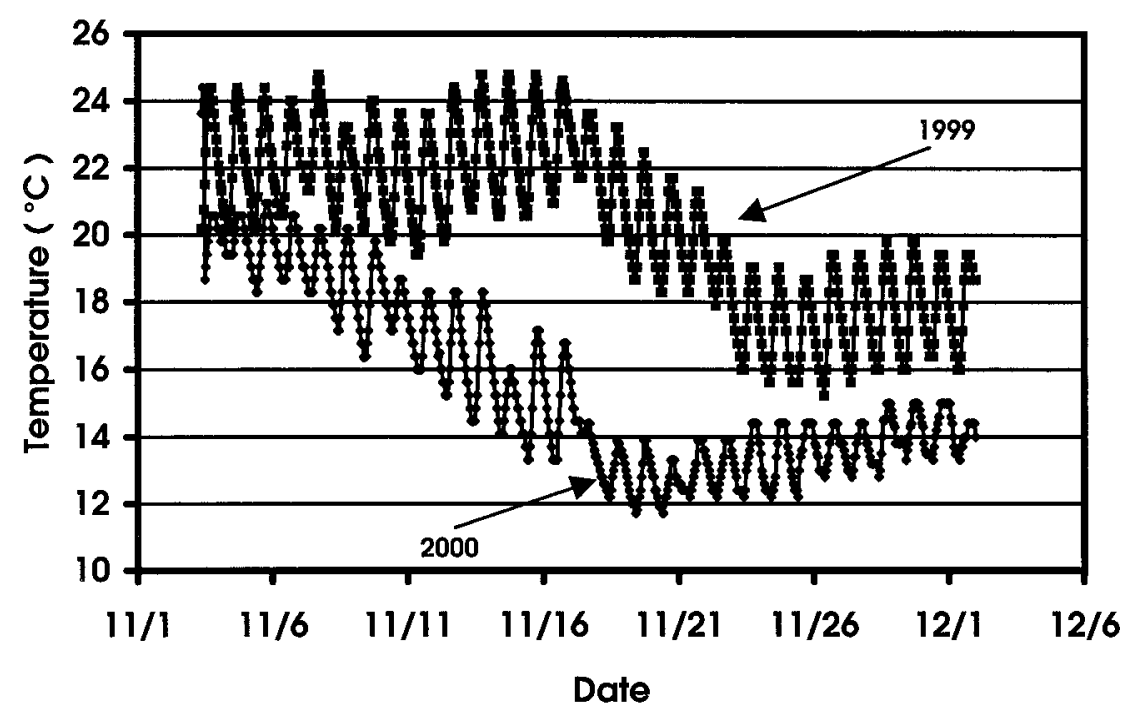

Fig. 3. Soil temperatures at the end of the 1999 and 2000 seasons for fall-planted cantaloupes in a commercial field in Winterhaven, CA. Data points represent hourly high and low temperatures at the 10 -cm depth.

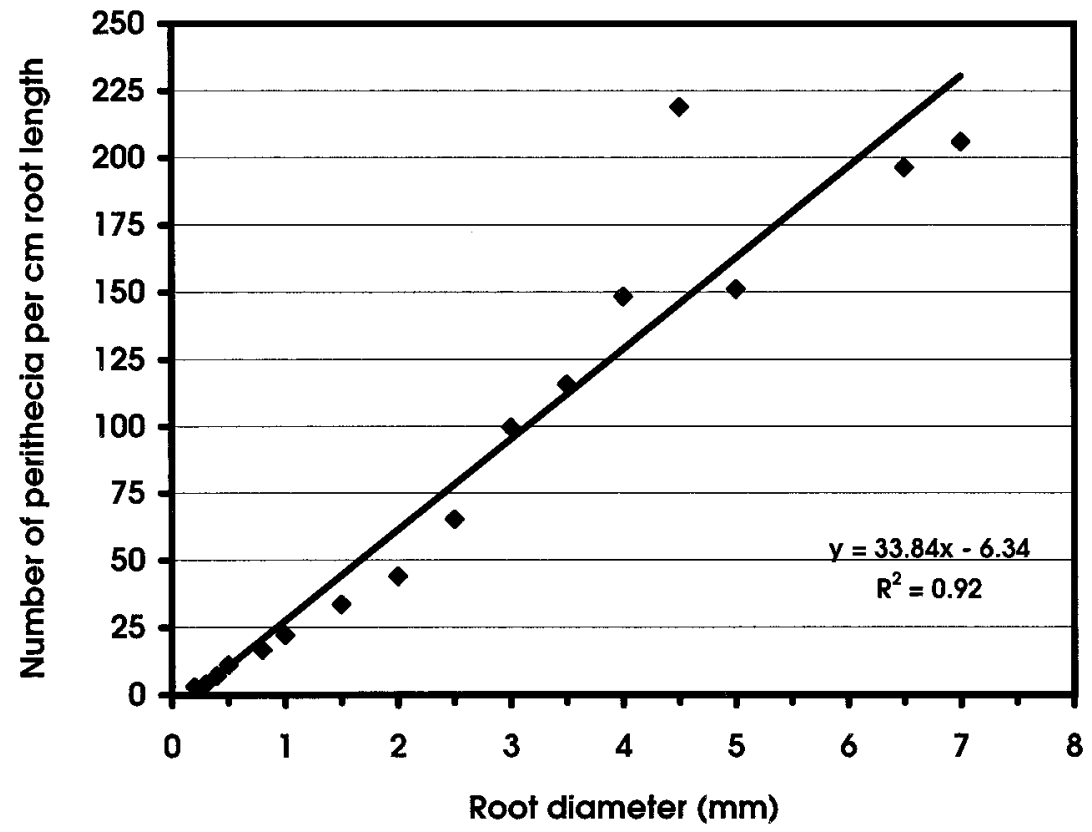

Fig. 4. The relationship between cantaloupe (Caravelle) root diameter and the number of perithecia of Monosporascus cannonballus per $\mathrm{cm}$ of root length at the end of the spring cropping seasons in 1999 and 2000. Values for means of observed data are represented by diamonds, and regression model is represented by a line $(P<0.0001)$. 
Table 1. Number of ascospores of Monosporascus cannonballus produced on root systems of four naturally infected cantaloupe plants

\begin{tabular}{lcccc}
\hline Plant number & $\begin{array}{c}\text { Number of } \\
\text { roots }^{\mathbf{a}}\end{array}$ & $\begin{array}{c}\text { Total root } \\
\text { length }(\mathbf{c m})\end{array}$ & $\begin{array}{c}\text { Root length with } \\
\text { perithecia (\%) }\end{array}$ & $\begin{array}{c}\text { Number of } \\
\text { ascospores }^{\mathbf{b}}\end{array}$ \\
\hline 1 & 15 & 141 & 100 & 504,000 \\
2 & 12 & 111 & 100 & 518,000 \\
3 & 16 & 144 & 100 & 266,000 \\
4 & 13 & 110 & 100 & 294,000 \\
Mean & 14 & $127 \pm 10$ & 100 & $395,500 \pm 66,989$ \\
\hline
\end{tabular}

a Taproots were not included in this study because there were no perithecia formed on them in the field at the time the plants were collected.

b Ascospores were enumerated as follows: roots were homogenized, and ascospores were separated from root debris by sieving and then suspended in a $50 \%$ sucrose solution. Ascospore populations in aliquots of the suspension were counted and the final number of ascospores determined based on volume of suspension.
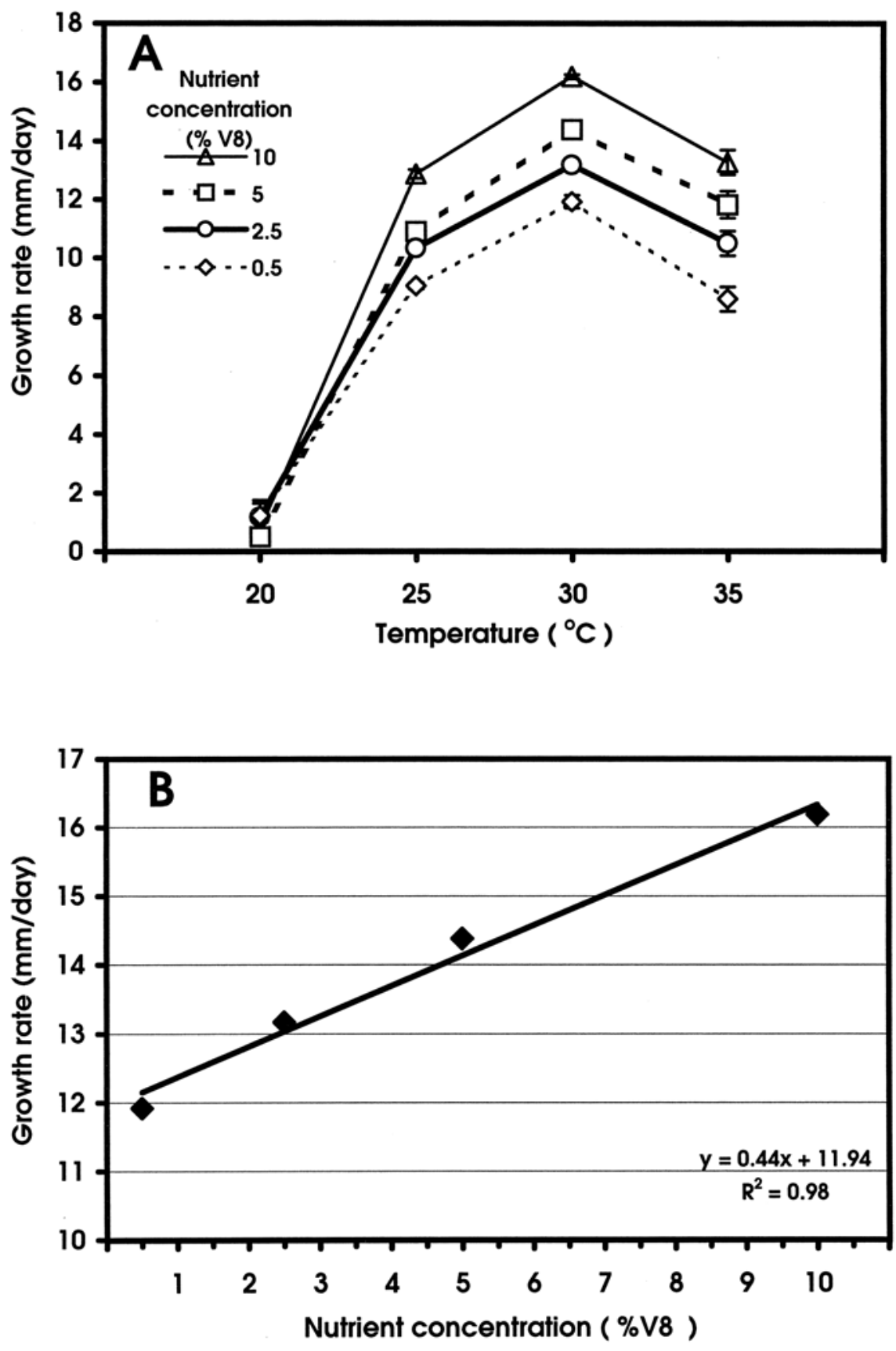

Fig. 5. Effects of temperature and nutrient concentration on the rate of mycelial growth of Monosporascus cannonballus. A, Effect of temperature on the growth rate at $0.5,2.5,5$, or $10 \%$ V8 juice agar. B, Relationship between nutrient concentration and mycelial growth rate at $30^{\circ} \mathrm{C}$. Combined results of two separate experiments, representing a total of six replicates per treatment, are shown. Vertical bars represent the standard error of the mean. Error bars within symbols are not shown. numbers of perithecia formed at $25^{\circ} \mathrm{C}$ $\left(45.7 \pm 2.1\right.$ perithecia $\left./ \mathrm{cm}^{2}\right)$ and $30^{\circ} \mathrm{C}(43.0$ \pm 0.9 perithecia $\left./ \mathrm{cm}^{2}\right)$ (Fig. $\left.7 \mathrm{~A}\right)$. No mature perithecia formed at $20^{\circ} \mathrm{C}$, and few $(3.9 \pm$ 0.7 perithecia $\left./ \mathrm{cm}^{2}\right)$ were formed at $35^{\circ} \mathrm{C}$ (Fig. 7A). There was a positive linear relationship $\left(R^{2}>0.9\right)$ between nutrient concentration in the culture medium and the number of perithecia produced at 25, 30, and $35^{\circ} \mathrm{C}$. Regression analyses for all temperature data exhibited the same trend; thus only the data for $30^{\circ} \mathrm{C}$ are presented $\left(P=0.04, R^{2}=0.93\right)($ Fig. $7 \mathrm{~B})$.

\section{DISCUSSION}

We sought to determine the rate of reproduction as well as the reproductive potential of Monosporascus cannonballus, a root-infecting fungus, on cantaloupe roots under field conditions. In the desert agricultural production regions of California, there are two cantaloupe-cropping periods. The spring planting period is from February to April with harvest from May to early July, and the fall planting period is from late July to early August with harvest from mid-October to late November.

Results of our investigation provide quantitative evidence that, irrespective of the particular cropping period, pathogen reproduction (e.g., production of ascospores) occurs primarily late in the growing season and that conducive soil temperatures govern, in part, the rate (speed) of reproduction of the pathogen at the end of each growing season. Specifically, during the latter part of both the spring and fall growing seasons, perithecia were observed on a relatively small percentage of the roots (mean of 18\%). Within the next 7 to 14 days, however, up to a fourfold increase in the percentage of roots bearing perithecia was recorded. The rate of increase in the percentage of roots bearing perithecia was, in general, two to four times faster at the end of the spring compared with the end of the fall cropping season. Variation in the rate of pathogen reproduction between the spring and fall seasons, or between different years within the same season (i.e., comparison of the fall 1999 and 2000 cropping seasons), was attributed to differences in soil temperature, which, as documented in our in vitro temperature studies, directly affects pathogen reproduction. The optimum temperatures for reproduction of the fungus ranged from 25 to $30^{\circ} \mathrm{C}$, with no reproduction at $20^{\circ} \mathrm{C}$ or lower. Soil temperatures at the 10 $\mathrm{cm}$ depth ranged from a low of $27^{\circ} \mathrm{C}$ to a high of $34^{\circ} \mathrm{C}$ at the end of the spring cropping season, whereas soil temperatures at the end of the fall cropping season ranged from a low of $12^{\circ} \mathrm{C}$ to a high of $20^{\circ} \mathrm{C}$.

Concomitant with the increase in production of perithecia at the end of the growing season was an increase in the number of ascospores. As previously mentioned, ascospores are the primary, if not sole, survival structure and form of inocu- 
lum for this root-infecting, soilborne pathogen. Our data, obtained via two different assessment methods, indicated that the root system of a single infected cantaloupe plant was capable of supporting the production of ca. 400,000 ascospores. The latter ascospore population, if incorporated uniformly into $0.03 \mathrm{~m}^{3}$ (one $\mathrm{ft}^{3}$ ) of soil, would, based upon that soil volume multiplied by the bulk density of the soil (i.e., $1.36 \mathrm{~g} / \mathrm{cm}^{3}$ ), raise the pathogen population to ca. 10 ascospores per gram of soil. Commercial cantaloupe fields with a known history of disease caused by $M$. cannonballus contain as few as 2.0 ascospores per gram of soil. The reproductive capabilities of the pathogen, as documented in our investigation, thus provide an explanation for the increased prevalence/severity of the disease in commercial fields, particularly those that have been sequentially cropped to cantaloupe.

The reproductive potential, in addition to the specific time of reproduction (i.e., late in the growing season), is not unique to the Monosporascus-cantaloupe interaction. Macrophomina phaseolina, a soilborne pathogen of numerous crops, reportedly is capable of producing approximately 560,000 sclerotia in a single corn stalk (2), and up to 50,000 sclerotia of Verticillium dahliae Kleb. have been estimated in a 2.5cm-long segment of an infected potato stem (6). Additionally, both of these rootinfecting pathogens produce their sclerotia late in the growing season, usually following death of infected plant tissues $(6,9)$. In general, the induction of resting spore reproduction under field situations has been attributed primarily to the depletion of nutrients in infected host tissue. With respect to $M$. cannonballus, induction of sporulation under field conditions appears to coincide with root death, but whether that is attributable to decreasing nutrient levels in infected roots has not been established. We have consistently observed that small lesion-bearing roots (i.e., those $<1$ $\mathrm{mm}$ in diameter), compared with large lesion-bearing roots (i.e., those $>2 \mathrm{~mm}$ in diameter), were the first to bear perithecia under field conditions. Although it is logical to assume that nutrient depletion in small infected roots would occur before depletion in large infected roots, our pureculture studies indicated that there was no relationship between the rapidity of perithecia formation and the nutrient concentration of the growth medium. Mature perithecia were consistently observed after 20 days incubation, and maximum numbers were recorded after 27 days incubation in all nutrient concentrations of the growth medium and at all incubation temperatures. These latter results suggest that some factor(s) other than, or in addition to, nutrient depletion is associated with the induction of reproduction under field conditions. Studies to identify those factors are currently under investigation. Additionally,

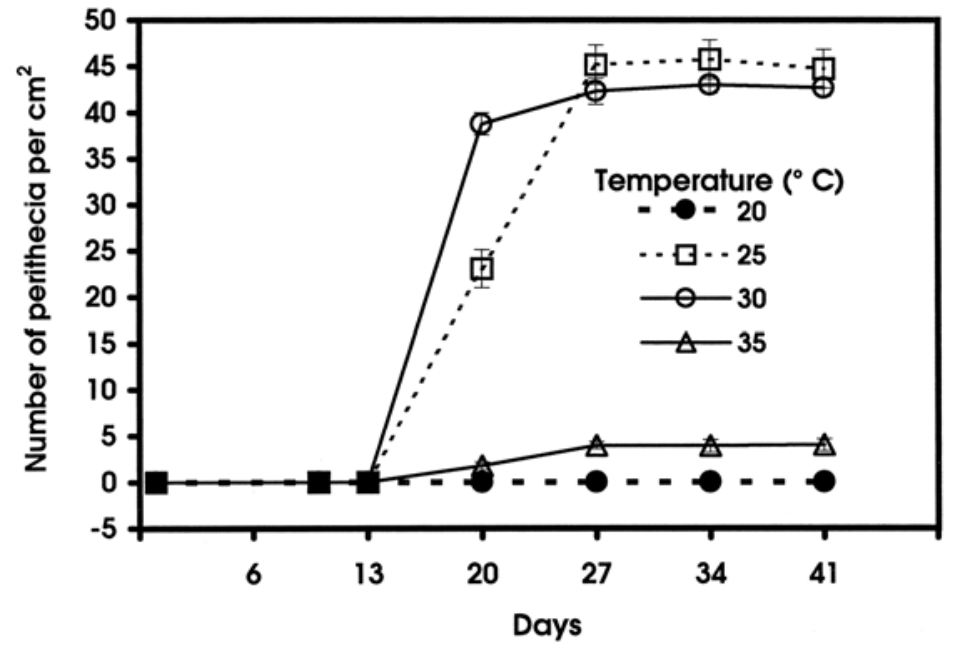

Fig. 6. Effect of temperature on progression of perithecia of Monosporascus cannonballus produced on $10 \%$ V8 juice agar. Combined results of two separate experiments, representing a total of six replicates per treatment, are shown. Vertical bars represent standard error of the mean. Error bars within symbols are not shown.
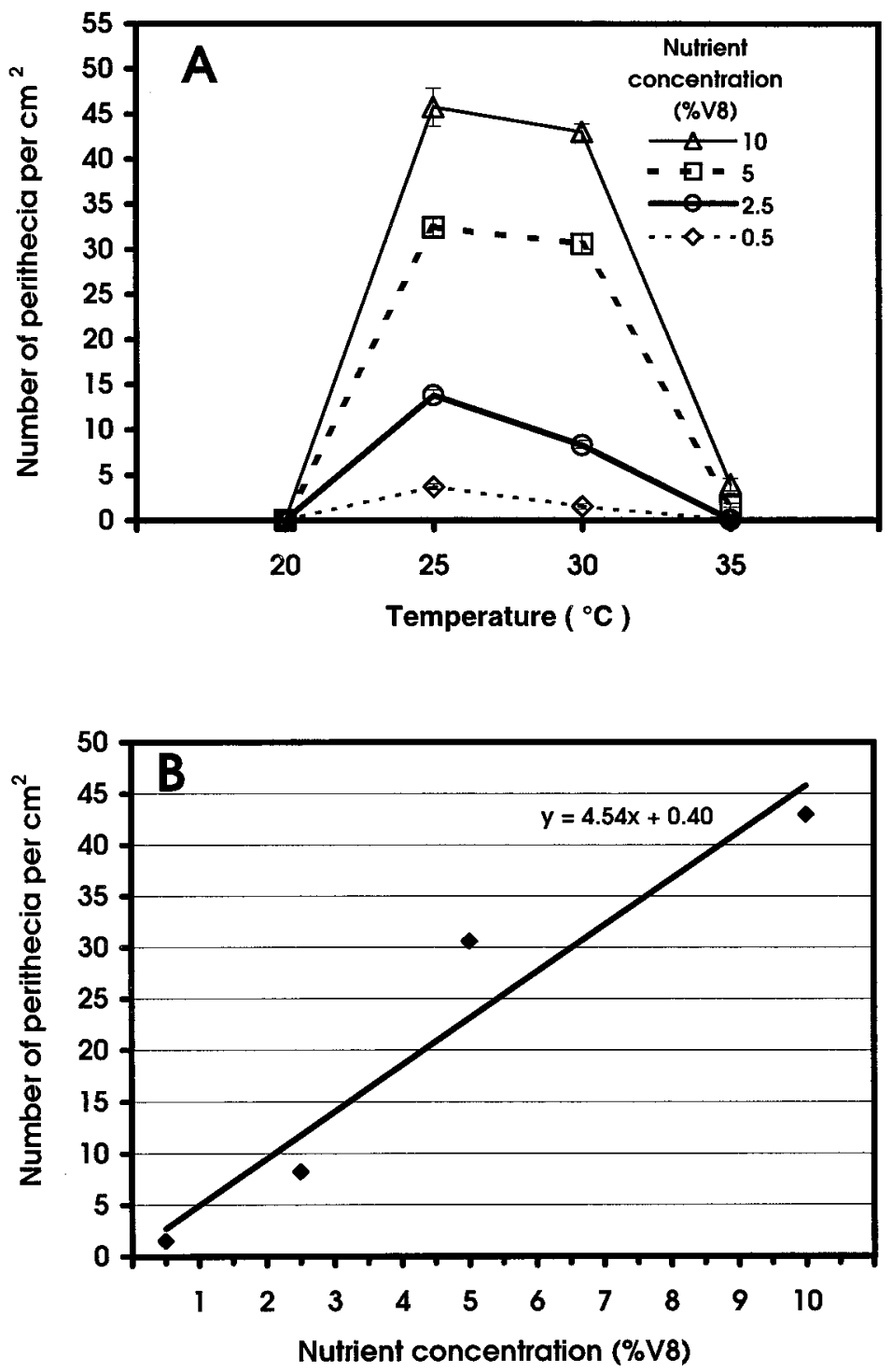

Fig. 7. A, Effects of temperature and nutrient concentration on number of perithecia produced in vitro. Vertical bars represent standard error of the mean. Error bars within symbols are not shown. B, Relationship between nutrient concentration and number of perithecia formed at $30^{\circ} \mathrm{C}$. Combined results of two separate experiments, representing a total of six replicates per treatment, are shown. Values for the means of the observed data are represented by diamonds and the regression model by a line $(P=0.035)$. 
the number of perithecia produced appears to be governed by root diameter, nutrients in that root volume, and soil temperature.

One of the objectives of studies involving the ecology and epidemiology of a plant pathogen is the identification of the "weak link" in the life or disease cycle. The results of our investigation suggest that the weak link in the life/disease cycle of Monosporascus cannonballus is located at the end of the cantaloupe growing season. Specifically, our results provide quantitative evidence that significant pathogen reproduction occurs primarily after the crop has been terminated. Thus, the key to maintaining low soil population densities of the pathogen resides in the inhibition of pathogen reproduction in infected roots left in the field after crop termination. Further, our results indicate that strategies to inhibit reproduction need to be implemented as soon as possible after crop termination, because significant pathogen reproduction occurs within 1 to 2 weeks after crop termination. Evaluation of the efficacy of several postseason root destruction methods for disease management (8), coupled with preplant strategies that reduce existing inoculum levels in soil (12), are currently under investigation.

\section{LITERATURE CITED}

1. Cohen, R., Pivonia, S., Burger, Y., Edelstein, M., Gamliel, A., and Katan, J. 2000. Toward integrated management of Monosporascus wilt of melons in Israel. Plant Dis. 84:496505.

2. Cook, G. E., Boosalis, M. G., Dunkle, L. D., and Odvody, G. N. 1973. Survival of Macrophomina phaseoli in corn and stalk residue. Plant Dis. Rep. 57:873-875.

3. Kim, D. H., Rasmussen, S. L., and Stanghellini, M. E. 1995. Monosporascus cannonballus root rot of muskmelon: Root infection and symptom development in relation to soil temperature. (Abstr.) Phytopathology 85:1195.

4. Martyn, R. D., Lovic, B. R., and Miller, M. E. 1995. Monosporascus root rot/vine decline of melons-A case study. Pages 36-43 in: Proc. Cucurbitaceae 94: Evaluation Enhancement Cucurbit Germplasm. G. E. Lester and J. R. Dunlap, eds. Gateway Printing, Edinbourgh, TX.

5. Martyn, R. D., and Miller, M. E. 1996. Monosporascus root rot and vine decline: An emerging disease of melons worldwide. Plant Dis. 80:716-725.

6. Menzies, J. D. 1970. Factors affecting plant pathogen populations in soil. Pages 16-21 in: Root Diseases and Soil-Borne Pathogens. T. A. Tousson, R. V. Bega, and P. E. Nelson, eds. University of California, Berkeley.

7. Miller, M. E., Martyn, R. D., Lovic, B. R., and Bruton, B. D. 1995. An overview of vine decline diseases of melon. Pages 31-35 in: Proc. Cucurbitaceae 94: Evaluation Enhancement Cucurbit Germplasm. G. E. Lester and J.
R. Dunlap, eds. Gateway Printing, Edinbourgh, TX.

8. Radewald, K. C., Stanghellini, M. E., Kim, D. H., Waugh, M. M., Mayberry, K. S., and Turini, T. 2001. Vine-decline of melons caused by Monosporascus cannonballus: III. Postharvest disease management strategies. (Abstr.) Phytopathology 91:S74.

9. Sewell, G. W. F. 1959. Direct observation of Verticillium albo-atrum in soil. Trans. $\mathrm{Br}$. Mycol. Soc. 42:312-321.

10. Stanghellini, M. E., Kim, D. H., and Rasmussen, S. L. 1996. Ascospores of Monosporascus cannonballus: Germination and distribution in cultivated and desert soils in Arizona. Phytopathology 86:509-514.

11. Stanghellini, M. E., Kim, D. H., and Waugh, M. 2000. Microbe-mediated germination of ascospores of Monosporascus cannonballus. Phytopathology 90:243-247.

12. Stanghellini, M. E., Kim, D. H., Waugh, M M., Radewald, K. C., Sims, J. J., Ohr, H. D. Mayberry, K. S., Turini, T., and McCaslin, M. A. 2001. Vine-decline of melons caused by Monosporascus cannonballus: I. Preplant disease management strategies. (Abstr.) Phytopathology 91:S84.

13. Stanghellini, M. E., and Rasmussen, S. L. 1992. A quantitative method for the recovery of ascospores of Monosporascus cannonballus from field soil. (Abstr.) Phytopathology 82:1115.

14. Waugh, M. M., Stanghellini, M. E., and Kim, D. H. 2000. Population dynamics of Monosporascus cannonballus. (Abstr.) Phytopathology 90:S82. 